\title{
Suicide « à la kashinawa ». Le désir de l'au-delà ou la séduction olfactive et auditive par les esprits des morts
}

\section{Barbara Keifenheim}

\section{(2) OpenEdition \\ Journals}

Édition électronique

URL : http://journals.openedition.org/jsa/2753

DOI : $10.4000 /$ jsa. 2753

ISSN : 1957-7842

Éditeur

Société des américanistes

Édition imprimée

Date de publication : 1 janvier 2002

Pagination : 91-110

ISSN : 0037-9174

\section{Référence électronique}

Barbara Keifenheim, « Suicide « à la kashinawa ». Le désir de l'au-delà ou la séduction olfactive et auditive par les esprits des morts", Journal de la société des américanistes [En ligne], 88 | 2002, mis en ligne le 05 janvier 2007, consulté le 19 avril 2019. URL : http://journals.openedition.org/jsa/2753 ; DOI : 10.4000/jsa.2753 


\title{
SUICIDE À LA KASHINAWA. LE DÉSIR DE L'AU-DELÀ OU LA SÉDUCTION OLFACTIVE ET AUDITIVE PAR LES ESPRITS DES MORTS ${ }^{1}$
}

\author{
Barbara KEIFENHEIM *
}

La conception kashinawa du suicide, selon laquelle la cause de la mort volontaire doit être recherchée dans un contact olfactif et auditif avec les esprits des morts, pose la question fondamentale du processus de médiation - spécifique à chaque culture - entre perception sensorielle et interprétation. Afin d'éclairer ce processus dans le contexte kashinawa, l'article présente quelques particularités de l'univers perceptif indigène.

Mots CLÉs : Amazonie péruvienne, Kashinawa, anthropologie des sens, suicide, perceptions sensorielles, esprits.

The Kashinawa's concept of suicide. Longing for the other world or sensory seduction by the spirits of the deceased

The Kashinawa's concept of suicide according to which the cause for a voluntary death must be searched for in the olfactory and auditory contact with the spirits of the deceased, raises fundamental questions concerning the process of mediation - specific to every culture between sensory perception and interpretation. In order to clarify this process in the case of the Kashinawa, this article presents some particularities of the indigenous universe of perception.

Key words : Peruvian Amazon, Kashinawa, anthropology of the senses, suicide, sensorial perceptions, spirits.

Suicidarse en el mundo kashinawa. El deseo del más allá o cómo los espiritus de los muertos seducen por los olores y los sonidos a los vivos

La concepción kashinawa del suicidio revela que la causa de toda muerte voluntaria se encuentra en un contacto olfativo u auditivo con los espíritus de los muertos. Esto nos remite al tema fundamental de los procesos de mediación - particulares a cada cultura — entre percepción sensorial e interpretación. Con el fin de dilucidar estos procesos en el contexto

* Équipe de recherche en ethnologie amérindienne, UPR 324 (CNRS), B.P. n 8, 7, rue Guy-Môquet, 94801 Villejuif cedex.

Journal de la Société des Américanistes, 2002, 88 : p. 91 à 110. Copyright $\bigcirc$ Société des Américanistes. 
kashinawa, el presente trabajo examina algunas particularidades del universo indigena de la percepción.

Palabras claves: Amazonia peruviana, kashinawa, antropología de los sentidos, suicidio, percepción sensorial, espíritus.

\section{INTRODUCTION}

Les Kashinawa ${ }^{2}$, qui sont environ au nombre de 5000 , appartiennent à la famille ethnolinguistique pano et vivent aujourd'hui dans la région frontalière séparant le Brésil et le Pérou ${ }^{3}$. Historiquement, les Kashinawa brésiliens et péruviens ont une origine territoriale commune : le cours supérieur du Río Murú et de ses affluents, le Río Iboiçu notamment, territoire qui appartient aujourd'hui à l'État brésilien d'Acre. Dans les années 1870, les barons du caoutchouc, brésiliens et péruviens, commencèrent à exploiter économiquement la zone. Les conséquences de la répression sur la population indigène furent catastrophiques et plusieurs groupes, dont les Kashinawa, cherchèrent refuge dans le bassin du haut Río Jurua. Cette région, cependant, ne demeura pas longtemps épargnée par l'exploitation du caoutchouc. Vers 1920, les Kashinawa se séparèrent quand une partie de l'ethnie dut fuir du Brésil vers la source du Río Curanja après l'assassinat d'un négociant de caoutchouc abhorré et le pillage de son dépôt ${ }^{4}$. La plupart des Kashinawa péruviens contemporains descendent de ce groupe qui évita, jusque dans les années quarante, tout contact avec les Blancs ainsi qu'avec d'autres indiens ${ }^{5}$. Aujourd'hui, ils sont intégrés dans la société péruvienne, mais profitant de leur isolement géographique, ils restent, comparativement à la branche brésilienne et à d'autres groupes indiens, relativement préservés d'influences exogènes massives ${ }^{6}$.

La chasse et l'agriculture sont les bases de l'économie kashinawa. Comme les plantations n'exigent que quelques semaines de travail durant la saison sèche, les hommes passent la majeure partie de leur temps à la chasse. La viande de gibier ne constitue pas seulement la nourriture privilégiée des Kashinawa, elle est aussi le bien d'échange le plus apprécié dans les relations sociales internes. Un bon chasseur étant en mesure de rapporter régulièrement plus de butin que sa famille ne peut en consommer, il peut alors en offrir aux autres et ainsi mettre en place un réseau étendu de relations privilégiées ${ }^{7}$. Cela accroît son prestige ainsi que son attrait sexuel aux yeux des femmes.

Le succès à la chasse ne dépend pas uniquement de l'habileté du chasseur, mais aussi et surtout de l'observation patiente de son territoire de chasse. C'est pourquoi les hommes kashinawa passent le plus clair de leur temps tout seuls dans la forêt profonde. À leur retour, lorsqu'ils franchissent le seuil du village, les chasseurs sont épiés avec autant de discrétion que d'attention. Certes, le premier regard sert à s'assurer si le chasseur rapporte du gibier sur son dos ou s'il revient bredouille, mais on guette également sa démarche et l'expression de son visage. S'il chancelle légèrement ou s'il a un regard absent, il suscite immédiatement l'inquiétude. On va l'entourer, le mener à son hamac et les femmes de sa maison vont susurrer anxieusement : « imana- $i$ - il est pris du désir de l'au-delà », et commencer tout de suite à préparer des remèdes destinés à écarter le mal menaçant. 
Que s'est-il passé à leurs yeux ? Pendant son passage dans la forêt profonde, certains esprits des morts ont at tiré le chasseur à l'aide de l'inin, une odeur envoûtante, et l'ont cerné de bruits et de sons qui pénètrent en lui. On croit que les odeurs et les sons sont accompagnés d'une substance invisible qui se pulvérise et se propage dans toutes les fibres du corps. Selon l'opinion commune, les contacts olfactifs et auditifs avec les esprits des morts conduisent inévitablement à la mort, si les premiers symptômes ne sont pas détectés et soignés à temps. Le traitement consiste, entre autres, à laver le patient avec une décoction d'herbes, à lui instiller le jus de plantes médicinales dans les yeux et à lui donner un remède qui lui fasse exsuder les substances entrées dans son corps. Ce faisant, on veille particulièrement à ce que personne ne se trouve à proximité du malade car, en pareil cas, les substances invisibles exsudées pourraient pénétrer dans un autre corps et y provoquer également le syndrome imana. Si aucun traitement n'est entrepris à temps, les progrès du mal sont décrits comme suit. D'abord, la victime des esprits des morts devient passive et tombe en léthargie. Elle va se plaindre de maux de tête violents, puis son corps entier sera en proie à de terribles douleurs. Avec le premier accès de fièvre, le désir de l'au-delà du patient devient de plus en plus fort. Et bien qu'il n'en ait encore eu qu'une « avant-odeur » attirante, il ne peut s'empêcher de penser sans cesse à cet au-delà. Le malade de l'imana commence à entendre et à sentir de manière "supra sensorielle ", puis il va voir les esprits des morts. Les membres défunts de sa famille vont lui apparaître, le toucher et l'inviter, à l'aide d'images fantastiques, à le suivre dans l'au-delà. Le malade refuse la nourriture humaine et demande à manger des vers et de la terre. Sa perception sensorielle ne fonctionne plus normalement : ses cinq sens sont tout entiers tendus vers l'au-delà. Ils ont perdu leur double vocation de sens d'être vivant et de sens d'être culturel, c'est-à-dire d'homme socialisé dans la communauté des Kashinawa. Le malade est en danger de mort parce qu'il ne peut plus opposer de vouloir-vivre à l'appel des morts. Il oublie ses liens familiaux et sociaux et ne répond plus aux appels de ses parents et amis. Dans peu de temps, il mourra.

Lorsque je racontai à mes interlocuteurs kashinawa de quelle manière certaines personnes de nos sociétés se donnent la mort, par exemple en se pendant, en se tirant une balle de revolver ou en se jetant du haut d'un bâtiment, ils furent très déconcertés et répondirent qu'il n'y avait chez eux qu'une seule manière de mettre fin à ses jours, imana. Quelqu'un qui succombe à la séduction olfactive et auditive des esprits des morts, me dirent-ils, finit par ne plus vouloir vivre, et par souhaiter la mort, «mawa katsis-ki».

La conception kashinawa du suicide, où s'exprime l'idée, étrange pour nous, que la cause de la mort volontaire doit être recherchée dans un événement sensoriel, pose la question fondamentale du processus de médiation - spécifique à chaque culture entre perception sensorielle et interprétation.

L'ethnologie a longtemps négligé l'étude des perceptions sensorielles et il a fallu attendre les années quatre-vingt avec les recherches notamment de Seeger (1975, 1981), Crocker (1985), Feld (1982, 1984, 1988), Stoller (1989), Howes (1991) et Classen (1993a, b) pour qu'une « anthropologie des sens » puisse s'établir. Je me sens fortement inspirée par deux objectifs de cette nouvelle sous-discipline ethnologique puisqu'ils permettent souvent de jeter une lumière nouvelle sur des questions classiques de la recherche amazonienne: d'une part, l'anthropologie des sens essaie de 
mettre en lumière le rapport complexe entre les sens de la perception et les constructions culturelles de réalité (Howes 1991, 1992 ; Synnott 1993 ; Classen 1993 ; Taussig 1993) ; d'autre part, elle vise à déduire de l'analyse des univers perceptifs indigènes des instruments descriptifs et analytiques par rapport à des phénomènes culturels difficilement accessibles à l'observateur occidental. C'est fréquemment le cas lorsque ce dernier est confronté avec une hiérarchie des sens qui, à la différence de la sienne, n'est pas dominée par le visuel. C'est également le cas quand on aborde le vaste domaine d'expressions performatives ou de situations liminales marquées par la suppression de l'ordre sensoriel quotidien des acteurs (Keifenheim 1999a, 1999b, 2000).

Afin de mieux comprendre le «suicide à la kashinawa », je proposerai donc une interprétation à partir d'une analyse de l'univers perceptif kashinawa et commencerai par présenter tout d'abord quelques concepts clés.

\section{LES PERCEPTIONS SENSORIELLES : ASPECTS DE LA CONCEPTION KASHINAWA ${ }^{8}$}

Le scepticisme des Kashinawa face à la fiabilité des perceptions sensibles est frappant. Cette position de principe est en rapport très étroit avec une conception du monde qui suppose l'existence de réalités multiples et interférentes ${ }^{9}$.

\subsection{La rupture de la création première}

Les mythes de création ${ }^{10}$ racontent qu'au commencement tous les êtres pouvaient communiquer entre eux et échanger leurs apparences extérieures à volonté (dami $\left.{ }^{11}\right)$. Le visible et l'invisible, le matériel et l'immatériel ne s'opposaient pas, mais étaient des phénomènes transformationnels fluctuants d'une seule et même réalité totale. Tout pouvait simultanément être soi-même et autre chose. Le principe de transformativité généralisée apparaît donc comme une caractéristique fondamentale de la création première et, figurant dans beaucoup de mythologies amazoniennes, il n'a pas échappé à l'attention des chercheurs. Ce qui peut-être a été bien moins mis en lumière est le fait que l'idée de mutabilité universelle va souvent de pair avec celle d'une disposition sensorielle spécifique. En fait, lorsque les Kashinawa racontent que des acteurs mythiques « se glissent dans la peau d'un autre », ils évoquent souvent explicitement que cette métamorphose, ou plutôt cette interpénétration des êtres par métamorphose, est accompagnée de modifications perceptives. Pour pouvoir communiquer et interagir en franchissant les frontières qui les séparent, les êtres originels « modifient » (dami) leur mode de perception. De cette manière, les différents ordres sensoriels se fondent en un seul, domnant, au moins provisoirement, une sphère de perceptions convergentes. On peut alors manger la même chose, s'accoupler et se reproduire, mais on peut surtout communiquer dans la même langue. Tout comme la capacité de modifier son mode de perception, la participation de tout le corps aux sensations visuelles est une caractéristique essentielle des perceptions « originelles». À cet égard, on retrouve dans les mythes de création des Kashinawa cette qualité de l'œil comme " organ of tactility » que Taussig a décrite comme caractéristique du concept de mimésis. Les perceptions visuelles mènent sans cesse à une " palpable, sensuous connection between the very body of the perceiver and the perceived » (Taussig 1993, p. 20 sq.). La fusion perceptive du soi et de l'autre dans les mythes de création libère un potentiel créateur par lequel de 
nouveaux êtres sont créés ou de nouveaux biens culturels voient le jour. Ainsi, la relation amoureuse entre un anaconda et un tapir ayant pris forme humaine donne naissance à l'art ornemental dans le monde aquatique souterrain. De même, la connaissance des drogues hallucinogènes est née des amours entre un ancêtre kashinawa et un anaconda ${ }^{12}$.

À la suite d'un incident désastreux, un processus de différenciation a eu lieu mettant fin à la création première. Depuis, le matériel et l'immatériel, le temps et l'espace, le visible et l'invisible sont des niveaux de réalité distincts. Pourtant, il n'y a aucune dichotomie absolue, les frontières sont extrêmement fluctuantes et des interférences peuvent jouer à tout moment.

À cause de la différenciation, chaque être vivant a désormais un corps (yuda) conforme à son espèce qu'un ou plusieurs esprits (yushin) animent, et il doit se contenter de rester " à l'intérieur de lui-même » durant la brève durée de son existence. Cette limitation est d'autant plus radicale qu'il a perdu la capacité de communication universelle. Les animaux sont condamnés à pousser des cris et les hommes sont prisonniers d'un langage trompeur qui suscite plus de malentendus que de compréhension. D’après les Kashinawa, la mort et les maladies sont nées en même temps que la parole.

Ainsi, l'apparition simultanée de la langue, des maladies et de la mort, celle de la communication trompeuse et du problème de la réalité et de l'apparence sont dues à la rupture de la création première. La condition humaine implique dorénavant que toute existence se déroule dans un champ de tension dangereux où tout peut continuellement basculer entre l'illusion et la réalité. Quel rôle y jouent les perceptions sensorielles?

\subsection{Illusion ou réalité?}

Si la communication universelle des temps immémoriaux allait de pair avec un mode perceptif spécifique, le processus de différenciation met également fin à la capacité de perception illimitée. Celle-ci correspondait fondamentalement à la réalité une de la création originelle. La coexistence de réalités multiples et interférentes rend désormais toute perception sensorielle problématique puisqu'il n'y a aucune certitude sur le niveau de réalité du phénomène perçu.

Le danger des réalités interférentes guette surtout celui qui quitte le domaine protégé du village et pénètre dans les profondeurs de la forêt. On y rencontre des animaux qui sont les égaux de l'homme chasseur ou des shomani, c'est-à-dire des êtres dont le corps est faible et qui lui sont par conséquent physiquement inférieurs, mais spirituellement incomparablement supérieurs. Le chasseur court ici le risque de rencontrer des hommes étrangers qui en veulent à sa vie. Dans la forêt résident aussi des esprits des morts qui peuvent lui faire abandonner la vie en exerçant sur lui leurs séductions. Ils peuvent lui apparaître sous forme humaine, parfois aussi sous celle d'un jaguar ou d'un autre animal. Comment peut-on dès lors savoir si l'on a affaire à un animal ou à un esprit des morts ? Où commence l'apparence trompeuse, où bascule la réalité ? La forêt profonde est considérée comme l'espace par excellence où les situations se renversent, où les frontières entre apparence et réalité se mettent à fluctuer et où il suffit d'un rien pour qu'une perception sensible devienne illusion fatale. 
Les stimuli sensibles sont donc fondamentalement polysémiques parce qu'on ne peut jamais les ranger de façon absolue dans l'un des multiples niveaux de réalité qui existent dans la conception kashinawa. En s'inspirant de la terminologie sémiotique, on pourrait parler ici d'un déficit référentiel des signifiants sensibles. Un seul et même parfum enivrant, l'inin, que le chasseur peut percevoir au cours de ses pérégrinations dans la forêt, peut aussi bien provenir d'une plante déterminée, un arbre nommé sinkabin, que d'un esprit des morts qui veut lui insuffler le désir de l'au-delà décrit plus haut. Un écoulement de salive, remarqué lors d'un réveil nocturne, peut aussi bien venir de troubles de la digestion que d'un rapport oral qu'un esprit des forêts a eu secrètement avec le dormeur.

\subsection{La notion de bai, " chemin 》}

Les perceptions sensibles sont pour les Kashinawa toujours l'objet de doutes métonymiques incitant à s'interroger continuellement sur les liens entre le perçu et le non-perceptible qui est considéré - et cela complique les choses - comme étant également doté de facultés perceptives. L'être percevant se trouve alors constamment dans une situation de perméabilité énergétique et subit aussi l'influence du nonperceptible percevant. Certes, l'appareil sensoriel de l'homme lui donne un accès au monde extérieur, mais le monde à la fois perçu et imperceptible pénètre en lui par le même chemin.

Dans ce contexte, l'importance conceptuelle de la notion de bai devient évidente. La signification première est " chemin ", avec une forte connotation de mouvement ${ }^{13}$. Dans le domaine sensoriel, bai désigne le parcours que suit un stimulus pour parvenir de sa source sensorielle à l'être percevant. Les Kashinawa distinguent entre trajectoires « droites» (bai yushtu-ma) et «sinueuses» (bai yushtu), et les sources sensorielles correspondantes sont jugées différemment. Ainsi, un stimulus olfactif n'est considéré comme fiable et inoffensif que s'il parvient à l'organe récepteur suivant une trajectoire droite. Les trajectoires sinueuses sont toujours les indices d'une source sensorielle suspecte. C'est dans le domaine auditif que la distinction qualitative entre les différents " chemins » est la plus marquée. Les sons de toutes sortes, les notes de musiques, les paroles humaines, les cris d'animaux, bref, tous les stimuli auditifs sont associés à la trajectoire sonore spécifique (bana bai ou ninka bai) qu'ils suivent pour arriver à l'oreille réceptrice. À l'intérieur du village, les trajectoires sonores déterminent la qualité de la communication. Parler fort (hancha kushipa) est vivement désapprouvé, parce que la voix suit alors selon les Kashinawa une trajectoire ondulatoire, décrit des cercles de plus en plus petits autour de l'auditeur qui finit par devenir sourd et léthargique à force de se défendre contre l'entrée du son, ce qui peut alors conduire à un affaiblissement du vouloir-vivre. En revanche, des sons mélodieux, les propos agréables (hancha pepa) évoquent l'idée de trajectoires sonores droites qui relient les hommes directement d'oreille à oreille, les rendant heureux et sereins. Un brouhaha de voix humaines (hancha husi-a) ou de bruits indistincts (bana husi-a) est insupportable aux Kashinawa. Les bégaiements suscitent le malaise puisque les sons émis sont également associés à une trajectoire sinueuse. Personne ne s'étonnera donc d'apprendre que les sons qui jouent un rôle décisif lors de l'événement sensoriel déclenchant le désir de l'au-delà parviennent aux victimes suivant une trajectoire sinueuse (bai yushtu). 
Quant aux perceptions olfactives, les trajectoires courbes ou tortueuses sont tout aussi négativement connotées que dans le domaine auditif. On prête au parfum enivrant inin deux trajectoires olfactives possibles, traduisant ainsi son extrême ambivalence. À l'intérieur du village, sa trajectoire est droite et directe et il accroît l'attrait sexuel des habitants. Les fruits de l'arbre sinbakin, qui sont utilisés pour la confection de mélanges parfumés, diffusent également leur arôme selon une trajectoire directe. Mais si l'odeur provient d'un esprit des morts qui veut éveiller le désir de l'au-delà chez un vivant, elle parvient à la victime selon une trajectoire courbe, l'encerclant tout comme une voix trop forte avant de pénétrer dans son nez et de déclencher ses effets destructeurs.

La nocivité potentielle des stimuli devient plus manifeste encore dans l'idée, déjà évoquée à propos du désir de l'au-delà, que les impressions sensorielles peuvent transmettre des substances dangereuses. Surtout les voix, les attouchements, les odeurs et les regards d'autrui peuvent faire pénétrer dans le corps des substances dangereuses conduisant dans le cas extrême à la mort. Cependant, il existe aussi certaines autres substances nocives qui traversent les différentes sphères cosmiques et qui peuvent également pénétrer dans le corps humain, de préférence par le nez, les oreilles et la peau. Ces substances sensibles errantes, une fois entrées dans le corps, modifient l'ordre sensoriel et, par là, la personnalité de la victime. Ainsi, la substance yupa provoque chez l'homme des perceptions déficientes qui réduisent à néant ses facultés de chasseur, du jour au lendemain. Malgré tous ses efforts, il ne pourra ni flairer ni entendre le gibier et, si un animal se trouve par hasard dans son champ de vision, sa flèche ne pourra quand même pas l'atteindre. À cause des déficiences de sa perception, l'homme envahi par le yupa ne pourra donc plus remplir ses obligations de chasseur. En même temps que son prestige social, il perdra aussi tout attrait sexuel et tombera peu à peu dans une situation de marginal ${ }^{14}$.

La présence de la substance amère muka dans le corps est une caractéristique essentielle des chamans. Les Kashinava désignent le chaman par le mot de huni $m u k a-y a$ : un homme avec la substance muka. Contrairement au malheureux chasseur, ce dernier voit ses facultés sensorielles se démultiplier. Cela l'éloigne progressivement de la sphère habituelle des interactions humaines et le fait pénétrer dans celle des communications avec les animaux et les esprits. Dès lors, il ne pourra plus tuer de gibier, parce que les frontières entre animaux, hommes et esprits disparaissent de son univers perceptif. Comment pourrait-il tuer un tapir alors qu'il est capable de communiquer avec lui dans sa langue ou qu'il reconnaît en lui un parent décédé ? Certes, ses remarquables facultés perceptives contribuent au bien spirituel de la communauté car il est capable de communication et d'interaction transcendantes, mais en tant que producteur, il représente une perte pour la société. C'est pourquoi aucune famille ne se réjouit quand l'un des siens manifeste une vocation de chaman.

Un traitement intensif est nécessaire pour se débarrasser des substances cosmiques. Elles sont considérées comme indestructibles et il ne peut en conséquence s'agir que de les rejeter hors du corps. Comme lors du traitement contre le désir de l'au-delà, on fait donc particulièrement attention à ce que personne ne se trouve à proximité du malade car, aussitôt sorties du corps, elles pourraient pénétrer dans un autre. 


\subsection{Stratégies de perception}

Les doutes métonymiques et le danger d'une transmission de substances nocives conduisent toute perception sensorielle à un dilemme existentiel : la moindre action quotidienne peut sembler tellement surchargée de dangers potentiels et de la complexité extrême d'interprétations possibles qu'il faut les réduire à un niveau supportable pour vivre le monde comme ordre et non comme chaos. Dans ce contexte, les stratégies de perception, dont l'apprentissage fait partie de la socialisation de tout Kashinawa, reçoivent toute leur signification. La stratégie la plus simple consiste à se boucher les organes des sens concernés en cas de danger; c'est-à-dire lorsqu'il est possible que les différents ordres de réalité se chevauchent et s'interpénètrent. On se bouche le nez et les oreilles, on ferme les yeux, on préserve sa peau des contacts extérieurs. Le but de ces mesures est d'interrompre le mouvement circulaire d'énergie entre le sujet percevant, le perçu et l'imperceptible percevant. On les inculque aux enfants, dès leur plus jeune âge, quand un tel comportement s'impose. Si, par exemple, les adultes constatent au lever du jour la présence d'une étrange odeur d'esprit (yushin inin), les enfants savent déjà ce qui va suivre : on leur mettra du coton dans les narines qu'ils devront garder un bon moment. Les jeunes chasseurs sont exhortés sans cesse à prêter attention au moindre comportement anormal des animaux de la forêt. Si un animal pousse un cri autre que celui propre à son espèce, si on l'a correctement abattu d'une flèche mais qu'on ne le voit pas s'effondrer, s'il se déplace de manière inhabituelle ou dégage une odeur atypique, le doute n'est plus permis. En cas de singularités de ce genre, on a sans aucun doute affaire à un esprit, non à un animal. Il faut alors abandonner toute ambition cynégétique, détourner le regard, se boucher le nez de feuilles et courir au village aussi vite que possible.

Une autre stratégie consiste à combiner vue, ouïe et odorat afin d'éviter les illusions sensorielles et les erreurs d'interprétation qui en découlent, surtout dans la forêt profonde et au contact d'étrangers. De très nombreuses expressions kashinawa reflètent cette idée de combinaison intersensorielle. On dit par exemple : «Quand je sens une odeur tout en entendant, je sais (c'est-à-dire je ne me trompe pas) Ninka-kin en shete-ai en unan-ai ». On dit aussi : "L'oreille et l'œil reconnaissent de concert — pabinkin inum bedu unan-nepa-ki » ou bien : " Le nez et l'œil reconnaissent de concert - dekinin inum bedu unan-nepa-ki. »

En général, l'œil est considéré comme centre d'intégration des diverses informations sensorielles. Dans la forêt, "l'œil veut toujours voir ce qui a été senti et reconnaître de concert avec l'oreille - shete-kin bedu-wen bechi-mis-ki, bedu-wen imun pabinkin unan-nepa-ki ». Dès qu'une sensation n'est pas confirmée par les impressions visuelles, auditives et olfactives correspondantes, subsiste le danger que des esprits des morts ou d'autres forces invisibles soient à l'œuvre.

Il faut également combiner les informations visuelles, auditives et olfactives lorsqu'on se trouve en contact avec des Blancs afin de découvrir leur manière d'être et leurs intentions véritables derrière leur façade extérieure. On va donc, lors de la visite de commerçants ou de représentants d'institutions politiques régionales, non seulement écouter le contenu de leurs discours, mais aussi veiller à ne pas « quitter des oreilles » les caractéristiques de la voix de chacun et de mettre en relation son comportement général avec l'odeur des différentes parties de son corps afin de les 
interpréter ${ }^{15}$. C'est ainsi que la démarche maladroite, les gesticulations continuelles d'un missionnaire, mais surtout sa voix considérée comme trop forte contribuèrent à réduire considérablement la crédibilité de son message de salut : ses propos annonçaient amour, paix et Rédemption, tandis que sa voix le démasquait comme puben : les Kashinawa désignent par ce mot un être aussi bien antisocial qu'anti-culturel.

La complexité fondamentale de l'interprétation des perceptions sensorielles est cependant atténuée par le fait que les couches de signification, par nature multiples, ne doivent pas être toutes mobilisées partout ni à tout moment. Elles peuvent être mobilisées ensemble ou séparément selon la personne, la situation et les attentes individuelles. Le séjour en dehors de l'espace social, le contact avec des étrangers, des pratiques rituelles exigent des dispositions et des interprétations perceptives spécifiques. Un chaman, un chasseur, un jeune qui suit un parcours initiatique, une tisseuse de dessins labyrinthiques vont associer et dissocier difléremment. La totalité des couches de signification possibles constitue un puissant modèle du monde, mais sa complexité multidimensionnelle n'est sûrement vécue par la plupart des Kashinawa que dans des situations liminales. Afin de pouvoir faire face aux exigences pratiques de la vie quotidienne et, plus fondamentalement, de ne pas vivre cela comme étant dépourvu de sens, il faut le plus souvent soumettre la polyvalence des perceptions sensibles à une sorte de dissociation. Ainsi le chasseur kashinawa doit-il, lorsqu'il voit un animal sauvage, se fier au fait qu'il s'agit bien d'un animal, s'il veut seulement le tuer. Mais presque tous peuvent raconter des situations limites où ils ont dû abandonner la proie parce qu'il était impossible de ne pas y voir un esprit des morts incarné.

En résumé, on peut dire que dans l'univers perceptuel des Kashinawa l'homme est fondamentalement perméable à l'interférence de forces extérieures. L'idée de perméabilité marque également la notion de la personne chez les Kashinawa ce qui est exprimé entre autres dans leur représentation de la relation corps-esprit chez les humains. Avant d'aller plus avant dans l'analyse, il convient de présenter le concept yushin, " esprit».

\section{LE CONCEPT YUSHIN, « ESPRIT »}

Dans la vision du monde des Kashinawa, toute chose existante est constituée de matière et d'esprit. Les deux sont des phénomènes de la création et ne peuvent en aucun cas exister isolés l'un de l'autre ${ }^{16}$. La seule chose qui puisse varier dans les différentes manifestations de la vie est le rapport spécifique entre matière et esprit. Ainsi, les humains possèdent un corps (yuda) et plusieurs esprits (yushin), mais ils sont tout d'abord et avant tout corps. Les esprits exogènes aussi possèdent un corps, mais ils sont tout d'abord et avant tout esprit. Du fait de leur nature corporelle peu marquée, ils sont doués d'une très grande capacité de métamorphose. Comme Townsley l'a montré de manière convaincante à propos des Yaminawa (qui appartiennent également à la famille pano), c'est "through the idea of yoshi ${ }^{17}$ that the fundamental sameness of the human and the non-human takes shape, creating the space for animal transformation of the human and the attribution of mental and human characteristics to all aspects of nature » (Townsley 1993, p. 456). Le côté yushin inhérent à toute chose existante produit des liaisons métonymiques entre des domaines différents. Il ne s'agit 
pas de relations d'analogie ou de métaphore, mais de "substantive connections » (ibid.).

L'aspect immatériel de la vie est toujours désigné par un seul et même terme, yushin, qu'il s'agisse d'êtres humains, d'animaux, de plantes ou d'esprits. L'homme se distingue par une relation corps-esprit particulière : son corps est animé ou, comme disent les Kashinawa, habité (hiwe-a-ki) par au moins quatre esprits :

- l'esprit des excréments (pui yushin)

- l'esprit de l'urine (isun yushin)

- l'esprit des yeux (bedu yushin)

- l'esprit de l'ombre du corps (yuda baka yushin) ${ }^{18}$.

Tandis que les esprits des excréments et de l'urine sont surtout associés aux processus et fonctions corporels, les esprits des yeux et de l'ombre du corps déterminent de manière décisive la personnalité d'un homme, bien que différemment. Après la mort, ils sont capables d'intervenir dans les affaires des vivants ${ }^{19}$. Ici, je ne traiterai que de ces deux derniers esprits, puisqu'ils permettent de mieux comprendre le phénomène du désir de l'au-delà.

\subsection{L'esprit des yeux : bedu yushin}

L'esprit des yeux existe déjà à la naissance : sans lui le corps ne serait, d'après les Kashinawa, qu'un morceau de chair, nami ${ }^{20}$. Il est aussi souvent appelé yushin kuin. La catégorie classificatoire réflexive kuin indique qu'il s'agit de l'esprit le moins lié à la matérialité du corps : c'est en lui que se manifeste le propre de l'« être yushin » au plus haut degré. Malgré son caractère constitutif pour la vie et la formation de la personnalité, c'est un esprit "vagabond " ${ }^{21}$. Lié au corps de manière très lâche, il peut le quitter très facilement. Sa capacité à communiquer et interagir avec les autres esprits issus de la création le soumet également à leurs influences, ce qui le fait accéder à un grand savoir, mais peut également provoquer maladies et autres maux. Il quitte régulièrement le corps pendant les rêves, les maladies fiévreuses, les pertes de conscience et lors de la consommation de drogues hallucinogènes. Les images intérieures qui naissent de ces états de conscience altérée sont considérées comme les produits des perceptions multisensorielles du bedu yushin lors de son errance hors du corps. Grâce à son instabilité, cet esprit retrouve donc en partie les capacités de métamorphose de la création première ainsi que les facultés de perception originelles.

Comme Townsley l'a vu le premier, le bedu yushin est avant tout une instance de perception. Dans son analyse de l'esprit des yeux en tant que " seat of perception », cet auteur souligne deux particularités paradoxales: "The first is the faculty of perception which permeates the body and at the same time can float free of it. The second is of a perceiving and vital self, radically mutable, which can transform itself so as to participate in all non-human aspects of the world » (Townsley 1993, p. 455).

Quand la mort d'un homme approche, c'est le premier esprit à quitter le corps. Certes, on peut vivre un certain temps sans lui, mais on ne fait que végéter. L'esprit quitte le corps, disent les Kashinawa, parce qu'il succombe aux séductions passionnées de ses proches parents décédés. Ils lui apparaissent, le visage et le corps magnifiquement peints, et l'invitent à les suivre dans le pays des morts avec l'image du hamac richement orné qui lui est destiné. 
La description du destin dans l'au-delà éclaircit également le lien étroit entre perceptions multisensorielles et esprit des yeux. Les Kashinawa s'accordent pour dire que seul l'esprit des yeux peut respirer après la mort, qu'il est seul doué de la vue, de l'odorat et du goût. Toutes les impressions sensibles persistent, mais de manière très atténuée.

Si l'on suit le chemin des esprits des yeux dans l'au-delà, on remarque qu'ils s'en vont vers des zones cosmiques toujours plus lointaines. Cet éloignement spatial va de pair avec l'affaiblissement progressif des liens qui les rattachent aux vivants, ou, autrement dit, du souvenir réciproque. C'est pourquoi les esprits des morts sont appelés pendant les trois premières années yushin bena («nouveaux esprits ») ou encore mawa bena (« nouveaux morts ») : ils sont rongés par le souvenir et le regret des vivants tout comme ceux-ci sont rongés par le souvenir et le regret des morts (mamu). Pendant cette période, les esprits des yeux parcourent sans répit les forêts avoisinant les lieux où ils séjournaient de leur vivant. Ils prêtent souvent assistance aux vivants en cas de danger spirituel ou physique et les protègent des esprits de l'ombre du corps qui persécutent les vivants (voir plus bas). Parfois, s'ils ont omis de le faire de leur vivant, ils transmettent leur savoir à l'aide de rêves. Après que le souvenir réciproque s'est peu à peu effacé, que les vivants et les morts se sont résignés à la séparation, les bedu yushin quittent les territoires terrestres et pénètrent dans une sorte de ciel intermédiaire. Si auparavant les vivants et les morts restaient en contacts fréquents à l'aide de rêves ou de drogues hallucinogènes, ce type de communication devient de plus en plus rare. Après un certain temps, les esprits des yeux se rendront dans les régions cosmiques les plus éloignées et y vivront avec l'Inka ${ }^{22}$ pour toujours. Leur souvenir est devenu si faible qu'ils ne pensent plus à rien de terrestre et entrent tout entier dans leur nouvelle vie : la contemplation de l'Inka.

L'effacement du souvenir et du regret connaît pourtant une exception significative : les consanguins les plus proches, que les esprits des yeux attendent même dans le ciel Inka. Ils iront les chercher dès que les « nouveaux morts » restés plus proches des vivants les informeront de leur vouloir vivre faiblissant. C'est le père mort qui viendra chercher son fils, la mère sa fille... C'est seulement lorsque leur bedu yushin sera lui aussi parvenu au pays des morts que tout souvenir du monde des vivants et toute relation avec lui disparaîtront.

Il faut ici prêter une attention particulière au motif de la contemplation comme état ultime de l'au-delà. Le fait déjà mentionné, que le bedu y'ushin continue à voir; entendre, sentir, etc., après la mort, révèle son caractère multisensoriel, ce qui souligne encore sa qualité de « seat of perception ». Ce n'est qu'avec la dissolution définitive de tous les liens qui le retenaient à l'ici-bas que les différentes modalités de perception se confondent en une seule : la pure contemplation de l'Inka. On peut interpréter cette fusion d'impressions sensorielles différentes comme une synesthésie. Les sens de la perception, bien distincts dans la vie terrestre, subissent après la mort un processus de différenciation à rebours et se rapprochent ainsi de l'état indifférencié de la création originelle. Le destin dans l'au-delà serait par conséquent un retour des modes de perception sensorielle vers leur état originel ${ }^{23}$. Dans cette perspective, les processus de perception synesthésique, tels qu'ils ont lieu sous l'effet de la drogue nixi pae et tels qu'ils sont explicitement décrits par les Kashinawa ${ }^{24}$, revêtent une grande importance épistémique : les perceptions induites par les hallucinogènes permettent de s'approcher d'une manière corporelle et sensible de l'état premier de la création ${ }^{25}$. 


\subsection{L'esprit de l'ombre du corps: yuda baka yushin}

Le yuda baka yushin enveloppe le corps comme une peau invisible. Les hommes ne sont pas les seuls à en avoir une. C'est le cas de toute chose existante, animée ou inanimée. Grâce à l'esprit de l'ombre du corps, on ne perçoit pas seulement la nature corporelle ou matérielle d'un être ou d'un objet, mais également le rayonnement de sa présence. Le yuda baka yushin veille sur la santé et la vitalité de l'homme vivant en même temps qu'il en est l'indice ${ }^{26}$. Comme tout esprit, il est invisible et insensible au toucher, mais son absence provoque chez l'homme une modification de son aura, comme c'est le cas chez le mourant. L'ombre du corps, ainsi que l'image qu'on aperçoit de soi ou d'un autre dans le miroir, sont des manifestations visibles de cet esprit. L'esprit de l'ombre du corps est associé à l'état de veille de l'homme, à ses actions, pensées et paroles conscientes, au savoir acquis par expérience ${ }^{27}$, et à la fonction de mémorisation ${ }^{28}$. Il agit donc à un niveau de conscience et de connaissance spécifique, celui dans lequel on cherche à s'ancrer dans la réalité quotidienne, sans devoir avoir sans cesse la coexistence d'autres réalités à l'esprit. À ce niveau de conscience, il est par exemple possible de se reposer sur ses connaissances des caractéristiques de telle ou telle espèce animale sans devoir systématiquement penser qu'il peut aussi bien s'agir d'un esprit. À la différence de l'esprit des yeux, sa présence chez le nouveau-né est encore peu marquée. Son développement et sa vigueur dépendent de la croissance corporelle et des expériences faites à l'état conscient. Comme les expériences spécifiques des hommes et celles des femmes se situent dans des domaines différents, il se développe différemment d'après le sexe de l'individu ${ }^{29}$. Il se distingue donc fondamentalement de l'esprit des yeux, dont la force provient surtout des interactions avec d'autres esprits en dehors de l'état de veille et qui est en conséquence indépendant de la personne et de son sexe.

Alors que l'esprit des yeux se sépare aisément pour toujours du corps d'un homme à la mort de celui-ci, les yuda baka yushin rôdent dans la forêt, furieux d'avoir perdu leur corps, et essaient de renouer des liens avec les vivants. Le désir de l'au-delà provoqué par une séduction olfactive et auditive est mis à leur compte.

C'est seulement lorsque leur souvenir s'est complètement éteint qu'ils vont vivre dans des régions de plus en plus lointaines de la forêt et constituer le peuple des ni nawa yushin (littéralement : « étrangers-esprits de la forêt »). Les ni nawa yushin sont de loin considérés comme les esprits les plus puissants et les plus dangereux et sont redoutés par les chamans eux-mêmes. Un de mes interlocuteurs m'a souvent répété ce qu'un chaman lui avait appris de la migration dans l'au-delà des yuda baka yushin : ils se rendraient progressivement dans les villes des Blancs au pays du froid ${ }^{30}$.

\subsection{Esprits des morts et mémoire}

Comme on peut s'en rendre compte, le temps post mortem des esprits des yeux comme celui des esprits de l'ombre du corps est essentiellement déterminé par leurs souvenirs. Tant que ceux-ci sont encore vivants, ils restent tous en contact avec les humains. C'est seulement avec l'extinction de tout souvenir de l'ici-bas que commence le temps d'un au-delà définitif. Néanmoins, il s'agit chez les deux types d'esprits d'une forme différente de mémoire. Alors que chez l'esprit des yeux la « mémoire sociale» 
survit et se manifeste dans les souvenirs des personnes qu'ils ont connues, c'est le souvenir impersonnel de la nature corporelle des vivants qui demeure chez les yuda baka yushin. Mes interlocuteurs ont souligné à plusieurs reprises que les esprits de l'ombre du corps menacent et « importunent » (bika wa-kin) sans cesse les humains parce qu'ils ne peuvent se résigner à la perte de leur corps ${ }^{31}$ et que par ailleurs ils ont oublié les règles de la vie en communauté et ne font donc plus preuve d'aucun respect. « Ninka-is-bu-ma-ki - ils n'écoutent plus ». Le " souvenir obsessionnel » (Lagrou 1992-1993) du corps humain concerne avant tout la sexualité. Les yuda baka yushin désirent avoir des rapports sexuels avec les vivants ou les tuer pour les attirer dans l'au-delà. L'idée que les esprits des morts puissent avoir des rapports sexuels avec les humains est profondément présente dans la pensée kashinawa. Ainsi, la naissance d'un enfant difforme est toujours attribuée au rapport qu'un yuda baka yushin a eu avec la mère sans qu'elle le sache. Conformément à cette idée, un tel enfant est appelé « enfant d'esprit » (yushin bake) ${ }^{32}$. D'emblée, on reconnaît aux yushin bake des perceptions sensorielles extraordinaires attribuées à l'aide du « père esprit ».

\section{LA CONSCIENCE SHINAN}

Les développements précédents ont montré que les Kashinawa conçoivent la personne humaine comme très perméable. Un être humain est animé par des esprits dont certains sont extrêmement vagabonds, et ses facultés de perception le rendent vulnérable à des puissances ou forces interférentes.

Il faut donc se protéger afin de préserver l'intégrité de son individualité. Ce rôle est joué par shinan, la conscience. Shinan est un concept très complexe et peut être considéré comme la principale force d'intégration qui agit aux niveaux de la personnalité les plus divers et détermine les forces physique, intellectuelle et sociale de l'être humain. La description des multiples niveaux interdépendants où shinan agit dépasserait le cadre de cet article. Je vais donc me limiter aux aspects les plus importants pour notre sujet.

\subsection{Shinan et l'indivisibilitas de l'homme}

Le shinan veille pendant la durée de la vie à ce qu'esprits et corps restent liés ${ }^{33}$. C'est donc le fondement de l'« in-dividu ». Il doit surtout surveiller l'instable esprit des yeux et le reconduire sans cesse au sein du corps. C'est pourquoi la plupart des maladies sont expliquées par une défaillance du shinan et les traitements visent essentiellement à renforcer le shinan chez le patient. Un shinan fort, c'est-à-dire un shinan kushipa, n'entretient pas seulement l'équilibre de la relation entre corps et esprit(s), mais protège aussi le corps de l'intrusion d'esprits exogènes et du danger qu'ils pourraient faire courir à l'indivisibilitas de l'homme.

\subsection{Shinan comme instance d'intégration perceptive et cognitive}

De plus, le shinan coordonne et intègre les différentes impressions sensibles et opère la synthèse cognitive. " C'est le shinan qui unit la vision, l'odorat, le toucher et l'ouïe - Uin-kin, shete-kin, me i-kin, ninka-kin, dasi wa-ti-ki shinan-dan ». Conformé- 
ment à cette conviction, les Kashinawa disent aussi que les sensations ne permettent pas à elles seules de saisir et de comprendre : « Bien que l'œil voie, bien qu'on entende, sente, touche, ils [c'est-à-dire les organes des sens, B.K.] ne sont que des trous béants Bedu-n uin-bia-kin, shete-bia-kin, me-bia-kin, pepu shui-ki ». La coordination et l'intégration opérées par le shinan sont nécessaires pour que des perceptions sensibles fassent sens. "Elles nous renseignent de concert - Meste wa-tan tapin-ti-iki». "Notre shinan leur procure la connaissance - Nukum shinan shabakabi wa-ti-ki ». Mais en cas d'un shinan faible, c'est-à-dire en cas de shinan babu, on devient facilement la victime des illusions des sens. Ainsi, les Kashinawa disent aussi : « Lorsque le shinan de quelqu'un s'est modifié, il amène l'œil à voir autrement - Hawen shinan dami-a bedu-an betsa wa i-mis-ki ». Si le shinan ne remplit plus sa fonction de surveillance, la voie est ouverte pour l'invasion de toutes sortes de yushin : « un homme sans shinam se trouve de ce fait si affaibli qu'il voit toujours des esprits (partout) - humi shinan-umadan haskai babu-shun yushin-bu uin-mis-ki ».

La faculté sensitive de l'esprit des yeux est, je l'ai déjà souligné, extrêmement ambivalente. Les sensations courantes sont accompagnées par d'autres sensations provenant du monde des esprits. On pourrait dire que l'esprit des yeux reçoit tous les stimuli sensoriels sans les ordonner en leur attribuant un sens. Par conséquent, il représente l'" état sensoriel brut ». Le shinan permet en revanche de faire la synthèse des perceptions sensorielles et ainsi de leur attribuer un sens.

\subsection{Shinan et la socialisation}

Comme le shinan n'existe à la naissance qu'en puissance ou, comme disent les Kashinawa, caché (bepu-ku-a), il faut l'éveiller, le renforcer au cours du processus de socialisation. D'après l'opinion commune, son développement est lié de façon essentielle à l'apprentissage de la langue. Sans la langue, pas de shinan. Dans ce contexte, les Kashinawa accordent une grande importance au nom (kena kuin) donné à l'enfant peu de temps après la naissance. Le kena kuin n'a pas de fonction vocative directe mais informe sur l'appartenance à un des huit shutabu (sections matrimoniales) ${ }^{34}$ permettant ainsi aux personnes de déduire leur relation précise qui sera énoncée à l'aide d'un vocable relationnel. Avant même que l'enfant ne soit capable de comprendre la complexité des différents types de relations sociales ainsi que les droits et devoirs spécifiques qu'ils impliquent, le nom permet de lui adresser la parole dès son plus jeune âge tout en évoquant par là son statut à l'intérieur de la société et de le familiariser à travers le langage avec les différenciations relationnelles de son entourage.

Avant de développer le shinan, l'enfant commence par parvenir à l'unan, le « savoir acquis par expérience ». Il finit par exemple par acquérir la maîtrise de son corps nécessaire pour pouvoir se nourrir lui-même. Il fait également l'expérience que tout ce que l'on prend dans sa bouche n'est pas mangeable. Mais il ne parvient au shinan dans le domaine de l'alimentation que lorsqu'il a compris et intégré que l'on partage la nourriture avec les autres en respectant certains critères de différenciation que rappellent entre autres les noms (kena kuin) des convives. Lorsqu'un enfant cesse d'être égocentrique (yaushi) et commence à se soucier des autres, tous se réjouissent du développement de son shinan. La conscience intégrative naît et s'affermit à travers les relations sociales, dont les normes sont transmises aux enfants par la parole et 
l'exemple des adultes. On cherche surtout à combattre les manifestations les plus diverses de l'avarice, comprise comme l'expression d'une fixation sur soi au détriment de la communauté. L'avarice peut s'exprimer dans les domaines de la vie les plus variés. Les hommes et les femmes sont considérés comme « avares de la langue » (hana yaushi) lorsqu'ils ne veulent pas parler aux autres, sont taciturnes ou ne prêtent pas attention à ce qui leur est dit. Ils sont « avares du pénis » (hina yaushi) ou « avares du vagin » (shebi yaushi) lorsqu'ils négligent sexuellement leur conjoint ou conjointe. Si les hommes ou les femmes ne veulent pas accorder à leur partenaire des amants ou maîtresses, on leur reproche leur " avarice d'amants » (ati yaushi). Les femmes passent pour être « avares d'enfants » (bake yaushi) lorsqu'elles refusent plusieurs grossesses. Les hommes et les femmes sont considérés comme mabu yaushi lorsqu'ils ne « veulent jamais rien prêter (inan katsi-is-ma-ki)». On leur fait grief d'« avarice de viande » (nami yaushi) lorsqu'ils ont de la viande chez eux et la mangent tout seuls au lieu d'inviter les autres. Les adultes pleinement socialisés incarnent en revanche avec leur beau shinan (shinan pepa) l'idéal de la vie heureuse, dont la description culmine toujours par l'énoncé des mêmes qualités normatives. Le shinan se développe et s'exprime de manière différente chez les hommes et les femmes. L'homme qui jouit d'un beau shinan, dit-on, s'acquitte au mieux des travaux dans ses plantations, est bon chasseur, n'est pas égoïste mais se soucie des autres, mène une vie heureuse et sans soucis, est en pleine santé. Toutefois, l'homme ne peut parachever son shinan que sur le plan spirituel, en apprenant les chants rituels (dewe). Une femme jouit d'un beau shinan quand elle s'acquitte de manière exemplaire de ses travaux domestiques, qu'elle est une hôtesse généreuse et se montre serviable avec tout le monde. La maîtrise de l'art ornemental est considérée comme le couronnement spirituel du shinan féminin. Les hommes et les femmes qui font preuve d'un shinan très développé sont des personnalités de grand renom et sont encore cités en exemple longtemps après leur mort. Quant à la faiblesse notoire du shinan, c'est-à-dire en fin de compte une socialisation ratée, elle se traduit par un comportement asocial ou par des bizarreries ${ }^{35}$. On éprouve généralement de la pitié pour une personne qui agit en contradiction avec les normes et les valeurs sociales, car cela montre que son shinan « s'est égaré » (benua-a-ki) et que l'intégration des divers niveaux physiques, sociaux et spirituels de l'existence ne peut plus avoir lieu.

\subsection{Défaillances de shinan}

Revenons-en au désir de l'au-delà, que les Kashinawa, lorsqu'on aborde le sujet en profondeur, mettent également en relation avec un shinan affaibli. Tout chasseur ne va pas être la victime de l'agression olfactive et auditive des yuda baka yushin. Tout le monde est au courant de l'ambivalence du parfum enivrant inin et connait les signes qui permettent de le classer, soit comme inoffensif et délicieux, soit comme dangereux. Ainsi, lorsque certains facteurs concordent, la perception d'une odeur inin doit être systématiquement interprétée comme la manœuvre agressive d'un esprit des morts : si la nuit tombe, qu'on se trouve seul dans la forêt profonde, et que l'intensité du parfum franchit un certain seuil alors qu'aucun arbre sinkabin, sa source naturelle, n'est présent. Connaissant ces facteurs conjoncturels, on peut prendre des mesures préventives qui assurent une protection presque certaine. Des gens incapables d'appliquer ce 
savoir lorsque la situation l'exige, on dit qu'ils ont, au sens propre du terme, l'esprit absent, qu'ils portent en eux quelque chose qui les tourmente et qui affaiblit leur attention envers leur environnement et les rend perméables à des influences exogènes. Il est intéressant de remarquer que ces défaillances sont le plus souvent attribuées à des conflits que nous qualifierions dans notre terminologie de psychosociaux. Les Kashinawa parlent simplement de tristesse ou d'accablement et les décrivent différemment selon le sexe. Ainsi, un homme est abattu (muitapa), quand les autres ne l'aident pas à faire son travail, ne l'invitent pas à manger; quand sa femme a des amants contre son gré, ne cuisine et ne lave plus pour lui, bref, lorsqu'il sombre dans l'isolement affectif et social. Une femme est nuitapa quand son conjoint a des maîtresses sans son accord, est paresseux, ne travaille pas à la plantation pour elle, et ne lui ramène pas le gibier convoité. Le désir suicidaire de l'au-delà montre de façon manifeste que le monde est ressenti comme hostile et il est en même temps un appel à la communauté afin que celle-ci remette les choses en ordre. Il est remarquable que les Kashinawa réagissent en effet au moindre symptôme d'imana. L'observation quasi routinière mentionnée au début doit être comprise ainsi, de même que l'anxiété qui germe dès que quelqu'un refuse toute nourriture et montre ainsi, au vrai sens du terme, qu'il n'a plus goût à la vie. Dès que l'on constate qu'une femme triste quitte régulièrement la maison en pleine nuit, on cherche à scruter l'expression de son visage. Si elle a l'air absent, on va craindre qu'elle se dirige vers la forêt pour chercher le contact avec les esprits des morts. Si l'on remarque qu'un homme ne travaille que pour lui, qu'il est taciturne ou qu'il ne paraît plus aux réunions quotidiennes, on conçoit également de l'inquiétude. C'est surtout le chef qui se sent investi de la responsabilité de découvrir ce qui tourmente un tel individu, car quelqu'un qui ne se sent pas en paix avec la communauté révèle que quelque chose ne va pas dans la société kashinawa. L'envie suicidaire est donc avant tout un problème communautaire, avant d'être un problème personnel. *

* Manuscrit reçu en avril 2002, accepté pour publication en juin 2002.

Traduit de l'allemand par Laurent Dedryvère.

NOTES

1. Je remercie le CNRS pour m'avoir offert un séjour de chercheur invité au sein du Laboratoire d'ethnologie et de sociologie comparative (UMR 7535, CNRS-Paris X). Durant cette période (janvier-avril 2000), j’ai pu analyser des données de terrain inexploitées jusqu'alors et rédiger une première version de cet article. Pour leurs conseils et suggestions, je remercie vivement mes collègues du Laboratoire et tout particulièrement Philippe Erikson. Je remercie également mes ami(e)s et collègues de l'EREA (UPR 324, CNRS) pour les chaleureux échanges d'idées.

2. L'auto-dénomination des Kashinawa est Huni Kuin. Puisque la plupart des groupes pano se dénomment ainsi (abstraction faite des variantes phonologiques), je préfère, en définitive, les appeler Kashinawa, terme généralement utilisé dans les publications internationales et permettant aux lecteurs de repérer facilement de quel groupe pano il s'agit dans mes écrits.

3. Pour la répartition géographique des groupes pano, leurs points communs et leurs divergences, $c f$. Erikson (1993, 1996) et Erikson et al. (1994).

4. Cf. McCallum (1990, p. 414) et Kensinger (1995, pp. 1, 265 sq.)

5. Sur le destin des Kashinasa du Brésil, cf. McCallum 1989 et 1990, Kensinger 1995, p. 2. 
6. Mes analyses concernent exclusivement les Kashinawa du Pérou auprès desquels je me rends régulièrement depuis 1977. Cf. Keifenheim 1990, 1992, 1996, 1997, 1999a, 1999b, 2000 et Deshayes \& Keifenheim 1994.

7. Cf. Deshayes 1992. Par rapport à la circulation de viande, on peut pourtant constater des changements importants depuis quelques années. Comme la viande de gibier constitue l'un des rares biens qui se prête à la vente aux métis du village frontalier de Puerto Esperanza, de nombreux Kashinawa cherchent à vendre leur butin de chasse aux commerçants de cet endroit. De ce fait, les dons de viande se font de plus en plus rares.

8. Pour une analyse détaillée, $c f$. Keifenheim 2000, pp. 69-158.

9. Cette conception est très répandue en Amazonie, $c f$. entre autres Gebhardt-Sayer $(1984,1986)$ et Illius (1988) pour les Shipibo-Conibo et, en dehors du contexte pano, Langdon (1992) pour les Siona, Guss (1989) pour les Yekuana, Taussig (1993) pour les Cuna et Gow (1988) pour les Piro.

10. Les Kashinawa partagent avec la plupart des autres groupes pano un très important fonds de motifs mythologiques communs.

11. Les « métamorphoses » désignées par dami sont assimilées par les Kashinawa à la créativité. Ce terme peut signifier que l'on souligne le passage d'un état ontologique à un autre, mais aussi que l'on met en avant le processus par lequel on transforme les matériaux bruts en produits. Les dessins d'objets que les enfants apprennent aujourd'hui à reproduire à l'école sont également appelés dami. La créativité consiste ici dans l'art de rendre présent quelque chose d'absent, c'est-à-dire de le re-présenter. D'autres images telles que les visions dues à la consommation de drogues hallucinogènes ou à la fièvre sont également appelées dami. On leur attribue en effet un grand potentiel créatif.

12. Voir aussi Camargo (1999).

13. Les plantations et les cours d'eau sont aussi appelés bai. Les lignes qui composent les motifs ornementaux des tissus et des tatouages, le chemin des esprits des morts ainsi que les liens entre les différentes sphères cosmiques également. La forme verbale $b a$ - (ikiki) signifie aussi bien "rendre visite " que " naître » ou " apparaître ». Le concept de transformation, fondamental chez les Kashinawa, est commun à ces champs sémantiques. Cf. McCallum 1991, p. 22, et Keifenheim 2000, pp. 108, 113, 123, 138, 149, 150 sq. et 177.

14. Cf. Deshayes 1992.

15. D'après plusieurs interlocuteurs, on craint particulièrement " l'odeur des mains » (me itsa) capable de transmettre des substances mortelles. « Nous avons toujours peur que les Blancs veuillent nous tuer - nawa humi mawa katsi ik-ama date-mis-ki. "

16. Cf. Kensinger (1995, pp. 207 sq.)

17. Yoshi correspond au terme yushin chez les Kashinawa. Si l'on fait abstraction de variantes phonologiques, ce terme apparaît dans tous les groupes pano.

18. Certains interlocuteurs ajoutent « l'esprit des dents » (sheta yushin) ou « l'esprit de la langue » (hana yushin), mais il n'existe aucune unanimité à leur égard. Il n'y a pas de consensus non plus sur l'existence d'un "esprit des rêves " (nama yushin) que quelques-uns considèrent comme un esprit autonome tandis que d'autres l'interprètent comme un aspect spécifique de «l'esprit des yeux ».

19. Cf. Kensinger (1995). Certains de mes interlocuteurs chez les Shipibo-Conibo pensaient que le nombre des esprits des morts augmente avec la durée de l'humanité et qu'ils polluent dangereusement l'atmosphère, finissant par provoquer un cataclysme. Cf. Gebhardt-Sayer (1987, p. 49). Je n'ai certes pas pu constater l'existence de cette idée avec la même intensité chez les Kashinawa, mais plusieurs personnes rendaient les esprits des morts, de plus en plus nombreux, responsables de la détérioration des conditions de vie.

20. D'après Townsley, les Yaminawa formulent une idée très analogue (1993, p. 455).

21. Voir entre autres McCallum (1991, p. 4) et Kensinger (1995, pp. 232 sq.) pour les Kashinawa et Towsley (1993, p. 455) pour les Yaminawa. Chez les Shipibo-Conibo, les caractères essentiels que les Kashinawa attribuent au bedu yushin semblent être répartis entre plusieurs esprits. À ce sujet, $c f$. Illius (1987, pp. 99 sq.).

22. L'Inka représente le héros culturel primordial de la mythologie kashinawa. Cf. Deshayes \& Keifenheim (1994), Kensinger (1995) et McCallum (1989, 1996).

23. Pour l'interprétation de l'au-delà comme retour à l'âge originel, $c$. McCallum : "The eye soul returns to mythic, perfect villages in the sky: There it lives eternally, remembering its living kin. It may interact with their wandering dream soul (nama yushin) " (1991, p. 4). Cf. aussi McCallum 1996, p. 454. Si mes analyses du domaine perceptif recoupent les analyses de McCallum, je nuancerais ses développements sur les souvenirs que l'esprit des yeux garde des vivants : ils ne subsistent que pendant les premiers temps après la mort.

24. La sensation synesthésique est, par exemple, décrite anisi : Ma mi-n bedu betsa-ki, dami inm inin shete $i$-dam, ce qui signifie littéralement : «si ton ceil inspire maintenant des visions et des odeurs, il se métamorphose (constamment) ». 
25. Cf. Keifenheim 2000, pp. 131-149.

26. Voir également Kensinger 1995, p. 209.

27. L'expérience modifie, d'après les Kashinawa, profondément le corps et s'y inscrit comme « savoir du corps » (yuda uman). Voir également McCallum (1991, p. 3, et 1996, p. 449), Kensinger (1995, pp. 237 sq.) et Keifenheim (2000, p. 104).

28. Townsley parle de convictions analogues chez les Yaminawa (1993, pp. 455 sq.).

29. Voir à ce sujet McCallum 1996, p. 449.

30. Lorsqu'un jeune homme mourut pendant mon séjour, ses derniers mots furent qu'il allait maintenant danser dans les villes des Blancs. La croyance en la migration des esprits de l'ombre du corps vers le territoire des Blancs permet de conclure à une affinité entre les Blancs et les esprits des morts.

31. Townsley évoque des conceptions semblables chez les Yaminawa qui nomment cet esprit diawaka : "The diawaka [...] after death clings to the flesh and the human world. It is said to be grief-stricken, disorientated and highly dangerous. The form of funerary rites is largely dictated by the need to placate this spirit, make it "lie down ", " cool its anger ", and finally banish it 》 (1993, p. 445).

32. Illius dit la même chose des Shipibo-Conibo (1987, p. 148).

33. Illius souligne aussi dans son analyse du concept de shinan chez les Shipibo-Conibo l'importance de l'intégration corps/esprits et définit le shinan comme « force vitale impersonnelle » (1987, pp. 102 sq.). Pour une interprétation du concept de shinan chez les Shipibo-Conibo, cf. également Gebhardt-Sayer (1987, pp. 186 sq.)

34. Les shutabu constituent des unités sociales engendrées par une triple division de la société kashinawa : en deux moitiés totémiques, en deux sexes et en deux groupes de générations alternées. Cf. Deshayes \& Keifenheim (1994, pp. 62-135).

35. D'après Bertrand-Ricoveri, les Shipibo-Conibo expliquent les phénomènes suivants par une faiblesse du shinan : « Un comportement séducteur répété, masculin et féminin, l'oubli par les enfants de préceptes ou conseils formulés par les proches, les hallucinations et l'exhibition des organes génitaux lors d'un état confusionnel, la fréquence des rêves érotiques, expriment à des degrés divers un dérèglement du shinan » (1994, p. 387).

\section{RÉFÉRENCES BIBLIOGRAPHIQUES}

Bertrand-Ricoveri, Pierrette, [1994]. — Vision Blanche/Vision Indienne. Traversée anthropologique d'une culture amazonienne : les Shipibo de l'Ucayali, thèse de doctorat d'État, université de Paris v, tome II.

CAmargo, Éliane, 1999. - « Yube, o homem-sucuriju. Relato caxinaua », Amerindia, 24, Association d'ethnolinguistique amérindienne, Paris, pp. 185-212.

Classen, Constance, 1993a. - Exploring the senses in history and across cultures, Routledge, London/New York.

—, 1993b. - Worlds of Sense, Routledge, London/New York.

Crocker, J. Christopher 1985. - Vital Souls: Bororo Cosmology, Natural Symbolism and Shamanism, University of Arizona Press, Tuscon.

Deshayes, Patrick, 1992. - «Paroles chassées. Chamanisme et chefferie chez les Kashinawa », Journal de la Société des Américanistes, LxxvIII (2), pp. 95-106.

Deshayes, Patrick \& Barbara Keifenheim, 1994. - Penser l'autre chez les Indiens Huni Kuin de l'Amazonie, L'Harmattan, Paris, 240 p.

ERıкson, Philippe, 1993. - « Une nébuleuse compacte : le macro-ensemble pano », L'Homme, 126-128, XXXIII (2-4), pp. 45-58.

-, 1996. - La griffe des aïux. Marquage du corps et démarquages ethniques chez les Matis d'Amazonie, Peeters-France, Paris/Louvain, 398 p. 
Erikson, Ph., B. Illius, K. Kensinger \& M. S. De Aguiar, 1994. - « Kirinkobaon Kirika ("Gringos' Books "). An Annotated Panoan Bibliography », Chantiers Amerindia, no 19 (1), Association d'ethnolinguistique amérindienne, Paris, 148 p.

Feld, Steven, 1982. - Sound and Sentiment, Birds, Weepings, Poetics, and Song in Kaluli Expression, University of Pennsylvania Press, Philadelphia.

—, 1984. — «Sound Structure as Social Structure », in : Ethnomusicology, xxvII, n 3, pp. 383409.

—, 1988. — « Sound as a Symbolic System : The Kaluli Drum », in : Explorations in Ethnomusicology. Essays in honor of D. P. McAllester, DMM 9, Harmony Park Press, Detroit, pp. 147-158.

Gebhart-SAyer, Angelika, 1984. - The Cosmos Encoiled: Indian Art of the Peruvian Amazon. Exhibition Catalog, Center for Inter-American Relations, New York, 32 p.

—, 1986. - « Una terapía estética : los diseños visionarios del ayahuasca entre los Shipibo », América Indigena, xLVI, pp. 189-218.

—, 1987. — « Die Spitze des Bewußtseins. Untersuchungen zu Weltbild und Kunst der ShipiboConibo » (Münchener Beiträge z. Amerikanistik, 21), Hohenschäftlarn, Klaus Renner, Munich, $400 \mathrm{p}$.

Gow, Peter, 1988. - «Visual Compulsion. Design and Image in Western Amazonian Culture ", Revindi, 2, Budapest, pp. 19-32.

Guss, David M., 1989. - To Weave and Sing. Art, Symbol, and Narrative in South Americam Rain Forest, University of California Press, Berkeley/Los Angeles/London, XIII+274 p.

Howes, David (ed.), 1991. - The Varieties of Sensory Experience. A Reader in the Anthropology of the Senses, University of Toronto Press, Toronto.

-, 1992. - The Bounds of Sense: An Inquiry into the Sensory Orders of Western and Melanesian Society, Ph. D. dissertation, Université de Montréal.

Illius, Bruno, 1988. - « Die Große Boa. Kunst und Kosmologie der Shipibo-Conibo », in : M. Münzel (ed.), Die Mythen sehen. Bilder und Zeichnungen vom Amazonas. Roter Faden zur Ausstellung, tome xv, Museum für Völkerkunde, Frankfurt/Main, pp. 705-735.

Keifenheim, Barbara, 1990. — « Nawa, un concept clé de l'altérité chez les Pano », Journal de la Société des Américanistes, LxxvI, pp. 79-94.

—, 1992. — « Identité et altérité chez les indiens Pano », Journal de la Société des Américanistes, LXXVIII (2), pp. 79-93.

—, 1996. — «Wir, die Weißen und die Wilden. Fremd- und Eigenbilder der KashinawaIndianer im Wandel », Historische Anthropologie, vol. 3, Böhlau, Köln/Weimar/Wien, pp. 383-398.

—, 1997. — « Futurs beaux-frères ou esclaves ? Les Kashinawa découvrent des Indiens non contactés », Journal de la Société des Américanistes, 83, pp. 141-158.

—, 1999a. - " Concepts of Perception, Visual Practice, and Pattern Art among the Cashinahua Indians (Peruvian Amazon Area) », Visual Anthropology, 12 (1), pp. pp. 27-48.

—, 1999b. — «Zur Bedeutung drogen-induzierter Wahrnehmungsveränderungen bei den Kashinawa-Indianern Ost-Perus », Anthropos, 94, pp. 501-514.

—, 2000. - Wege der Sinne. Wahnehmung und Kunst bei den Kashinawa-Indianern Amazoniens, Campus, Frankfurt/M, 264 p.

Kensinger, Kenneth, 1995. - How Real People Ought To Live. The Cashinahua of Eastern Peru, Waveland Press, Prospect Heights. 
Lagrou, Elsje Maria, [1992-1993]. - « Caminhos, Duplos e Corpos : a experiência visual entre os Kaxinawá ", ms.

LANGDON, Esther Jean, 1992. - «Alucinógenos: Fonte de Inspiração Artística. A cultura Siona e a expêriencia alucinógena ", in : Vidal, Lux (ed.), Grafismo Indigena. Estudos de Antropologia Estética, Studio Nobel-FAPESP-EDUSP, São Paulo, 296 p.

McCallum, Cecilia, [1989]. — Gender; Personhood and Social Organization Amongst the Cashinahua of Western Amazonia, Doctoral Dissertation, London School of Economics.

—, 1990. — « Language, Kinship and Politics in Amazonia », Man, pp. 411-433.

-, [1991]. - «Cashinahua (Huni Kuin) Death, Dying and Personhood » (paper presented at the 47th International Congress of Americanists, New Orleans).

—, 1996. — « Morte e pessoa entre os Kaxinawa », Mana, 2 (2), pp. 49-84.

SeEger, Anthony, 1975. - « The Meaning of Body Ornaments : A Suya Example », Ethnology, 14,3 , pp. 211-224.

-, 1981. - Nature and Society in Central Brazil : The Suya Indians of Mato Grosso, Harvard University Press, Cambridge, Ma.

Stoller, Paul, 1989. - The Taste of Ethnographic Things. The Senses in Anthropology, University of Pennsylvania Press, Philadelphia.

Synnotr, Anthony, 1993. - The Body Social. Symbolism, Self and Society, Routledge, London.

TAussig, Michael, 1993. - Mimesis and Alterity : A Particular History of the Senses, Routledge, New York.

Townsley, Graham, 1993. — « Song Paths. The Ways and Means of Yaminahua Shamanic Knowledge », L'Homme 126-128, XxxIII (2-4), pp. 449-468. 